\title{
Injectable Nanoelectrodes Enable Wireless Deep Brain Stimulation of Native Tissue in Freely Moving Mice
}

\author{
Kristen L. Kozielski ${ }^{1,2 \# *}$, Ali Jahanshahi ${ }^{3 \#}$, Hunter B. Gilbert ${ }^{1,4}$, Yan Yu ${ }^{1}$, Önder Erin ${ }^{1,5}$, David \\ Francisco ${ }^{1}$, Faisal Alosaimi ${ }^{3}$, Yasin Temel $^{3 *}$, Metin Sitti $^{1 * *}$. \\ ${ }^{1}$ Department of Physical Intelligence, Max Planck Institute for Intelligent Systems, Stuttgart, \\ Germany. \\ ${ }^{2}$ Department of Bioengineering and Biosystems, Institute for Functional Interfaces, Karlsruhe \\ Institute of Technology, Karlsruhe, Germany. \\ ${ }^{3}$ Department of Neurosurgery, Maastricht University Medical Center, Maastricht, The \\ Netherlands. \\ ${ }^{4}$ Department of Mechanical and Industrial Engineering, Louisiana State University, Baton \\ Rouge, LA, USA. \\ ${ }^{5}$ Department of Mechanical Engineering, Carnegie Mellon University, Pittsburgh, PA, USA. \\ $\#,+$ These authors contributed equally to this work. \\ *Correspondence to: Kristen Kozielski, k.koz@kit.edu. Metin Sitti, sitti@is.mpg.de
}

\begin{abstract}
Devices that electrically modulate the central nervous system have enabled important breakthroughs in the management of neurological and psychiatric disorders. Such devices typically have centimeter-scale dimensions, requiring surgical implantation and wired-in powering. Using smaller, remotely powered materials could lead to less invasive neuromodulation. Herein, we present injectable magnetoelectric nanoelectrodes that wirelessly transmit electrical signals to the brain in response to an external magnetic field. Importantly, this mechanism of modulation requires no genetic modification of the brain, and allows animals to freely move during stimulation. Using these nanoelectrodes, we demonstrate neuronal modulation in vitro and in deep brain targets in vivo. We also show that local thalamic modulation promotes modulation in other regions connected via basal ganglia circuitry, leading to behavioral changes in mice. Magnetoelectric materials present a versatile platform technology for less invasive, deep brain neuromodulation.
\end{abstract}




\section{Introduction}

Electrical communication with and modulation of the central nervous system (CNS) are essential to our current understanding of neurobiology, and in the diagnosis and treatment of neurological disorders. Using sensing and/or modulation of neural electrical activity, key therapeutic CNS interventions have allowed remarkable medical breakthroughs. For more than 30 years, deep brain stimulation (DBS) has provided patients with symptom relief from Parkinson's Disease, as well as other disorders, using electrodes wired into deep targets within the brain (1). More recently, closed-loop control of epidural electrical stimulation enabled walking in patients with spinal cord injury (2). Importantly, such devices function in freely moving patients, enabling daily activity and chronic patient use.

In recent years, efforts to make neural intervention less invasive, longer-lasting, and safer have progressed the capabilities of neural devices (for review, see (3)). A key challenge of such devices is powering, and wired-in powering can require that patients undergo surgical battery changes, every 3-5 years in the case of DBS devices (4). Instead, neural devices that are remotely powered have emerged using magnetic induction (5), opto-electric signaling (6-8), acoustic powering of piezoelectric materials (9-14), magnetic heating (15), piezoelectric powering of LEDs $(16,17)$, or magnetoelectric materials (18), instead of a wired-in battery.

Like conventional DBS electrodes, centimeter-scale devices require surgery and implantation of hardware external to the CNS, which risks brain hemorrhage, infection, and damage during daily activity (4). Thus, several neural device technologies have instead turned to smaller (nano- to millimeter-scale) devices, which can be completely implanted within the CNS, potentially via injection.

However, smaller size can make powering of neural devices more difficult. Remotely powered devices using magnetic induction (5), or opto-electric signaling $(6,7)$ thus far are limited in their tissue penetration depth, maximally reaching $1 \mathrm{~cm}$ and $6 \mathrm{~mm}$, respectively (19). Ultrasound-powered piezoelectric devices are perhaps the most promising of these technologies, recently showing recording at multiple sites through $5 \mathrm{~cm}$ of tissue phantom material with a sub$\mathrm{mm}^{3}$ device (10). Modulation with piezoelectric devices, however, has currently only been demonstrated in the peripheral nervous system using millimeter-scale devices, or in vitro (1214). As power transmission is typically done at the mechanical resonance frequency of such devices, this creates a fundamental tradeoff where an increasingly smaller device with a higher resonance frequency can be powered at increasingly shallower tissue depths $(20,21)$. Thus, resonant frequency signaling creates an obstacle to modulating deep brain targets with an injectable-sized device.

To circumvent signal transmission challenges, other strategies have used genetic neuronal modification and magnetic nanoparticles (15), or piezoelectrically-powered LEDs $(16,17)$ to trigger ion channel opening. However, the dependence of such technologies on genetic tissue modification creates regulatory barriers to their translation into patients. Wireless modulation of neural activity is clinically available using transcranial magnetic stimulation (TMS), which requires no implanted device (22). However, TMS only modulates cortical tissue (23), and has a depth-focal area tradeoff $(24,25)$, making DBS via TMS currently impossible.

To achieve wireless signal transmission to injectable devices, we have used magnetoelectric nanoelectrodes, which couple magnetic and electric signals (Fig. 1A). Technologies using magnetoelectric materials for neuromodulation have previously been 
explored. A centimeter-scale device has been used for DBS (18), and in other work, magnetoelectric nanoparticles were used but not reported to modulate activity in the deep brain (26). However, both demonstrate the promise of magnetoelectric materials for neural devices.

Herein, we report wireless DBS in vivo using injectable, magnetoelectric nanoelectrodes. They are implanted into the subthalamic area via stereotactic infusion, and stimulated using an external magnetic field at non-resonant frequencies, and in freely moving mice (Fig. 1C).

In particular, we made two-phase magnetoelectric nanoparticles (MENPs) using magnetostrictive $\mathrm{CoFe}_{2} \mathrm{O}_{4}$ nanoparticles (MSNPs) coated with piezoelectric $\mathrm{BaTiO}_{3}$. The two materials are strain-coupled via sol-gel growth of $\mathrm{BaTiO}_{3}$ on $\mathrm{CoFe}_{2} \mathrm{O}_{4}$ nanoparticles. Wireless particle stimulation is achieved by application of a magnetic field, which creates strain in $\mathrm{CoFe}_{2} \mathrm{O}_{4}$, resulting in applied strain to $\mathrm{BaTiO}_{3}$, thereby creating a charge separation (Fig. 1A). Below, we demonstrate wireless generation of an electric field across MENPs using an applied magnetic field. We then show that magnetic stimulation of MENPs enables wireless modulation of neuronal activity in vitro and in vivo. Finally, we demonstrate the therapeutic potential of this technology through its ability to modulate activity in the motor cortex and nonmotor thalamus, and to alter animal behavior.

\section{Results and Discussion}

Two-phase MENPs were synthesized using a protocol similar to Corral-Flores et al. (27). The nanoparticles were characterized for morphology (Fig. 1D,E), magnetostrictive to piezoelectric material ratio (Fig. 1E), and crystal structure (Fig. 1F). We observed two-phase MENPs containing $36.1 \pm 0.6 \% \mathrm{BaTiO}_{3}$ and $63.9 \pm 0.6 \% \mathrm{CoFe}_{2} \mathrm{O}_{4}$, in their perovskite and spinel crystal structures, respectively. MENP hydrodynamic properties were also characterized via dynamic light scattering (DLS) in cell culture medium and an artificial cerebrospinal fluid (aCSF) solution. Average particle diameter was measured as $224 \pm 17 \mathrm{~nm}$ and $277 \pm 18 \mathrm{~nm}$, and zeta potential was measured to be $-8.6 \pm 0.5 \mathrm{mV}$ and $-6.7 \pm 0.5 \mathrm{mV}$, in medium and aCSF, respectively (Fig. 1G).

We next measured the electrical output of MENPs under an applied magnetic field to characterize their ability to wirelessly generate an electric field. MENPs were measured as a sintered, poled pellet by attaching electrodes and measuring the output voltage via a lock-in amplifier (Fig. S1). A pellet containing only MSNPs was used as a negative control. To optimize our ME output, we applied a small AC magnetic field with a larger DC bias field along the same axis (Fig. 1B). The magnetoelectric coefficient $\left(\alpha_{M E}\right)$, which quantifies the relationship between the input AC magnetic field and output voltage, varied nonlinearly with the DC field, as has previously been reported (28). The $\alpha_{M E}$ reached a maximum of $27.6 \mathrm{mV} \mathrm{cm}^{-1} \mathrm{~T}^{-1}$ at 200 and 225 mT in the MENP pellet, while the MSNP $\alpha_{M E}$ showed no dependence on the DC field (Fig. 1H). Using a DC field within the maximum $\alpha_{M E}$ range $(220 \mathrm{mT})$, we measured a linear relationship between the AC field magnitude and the output voltage $\left(\mathrm{R}^{2}=99.8 \%\right.$ and $99.7 \%$ at $\mathrm{AC}$ frequencies of $140 \mathrm{~Hz}$ and $280 \mathrm{~Hz}$, respectively) (Fig. 1I), which is also typical of magnetoelectric materials (28). 

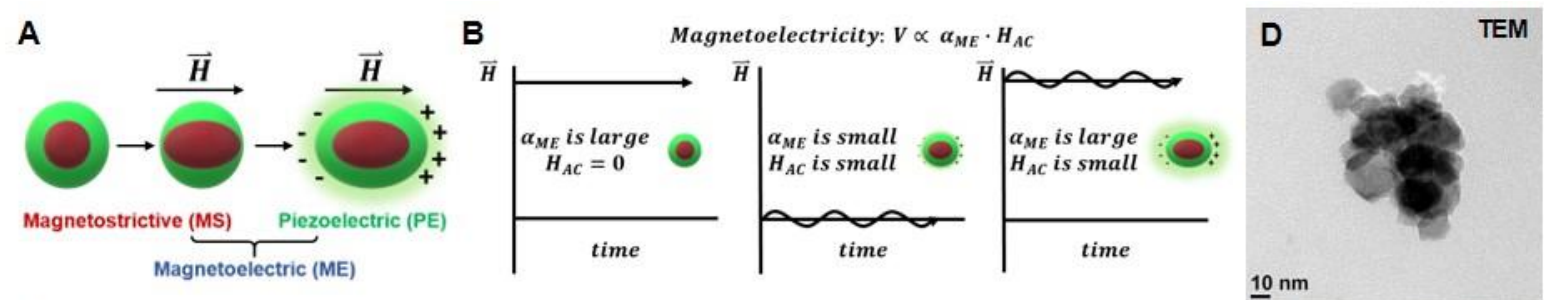

C

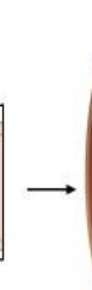

$\mathbf{F}$

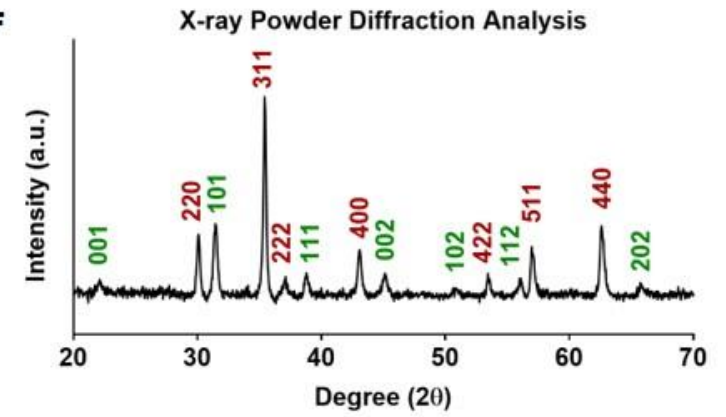

G

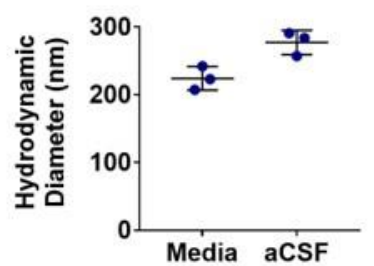

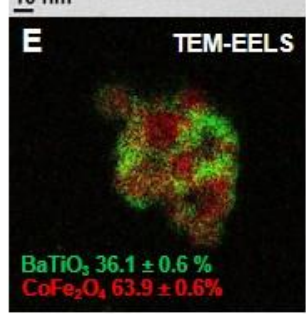

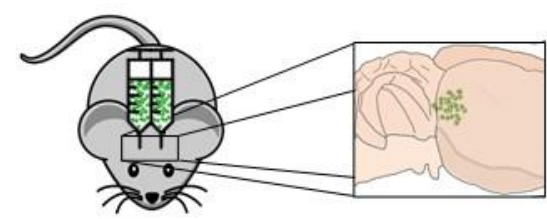

H

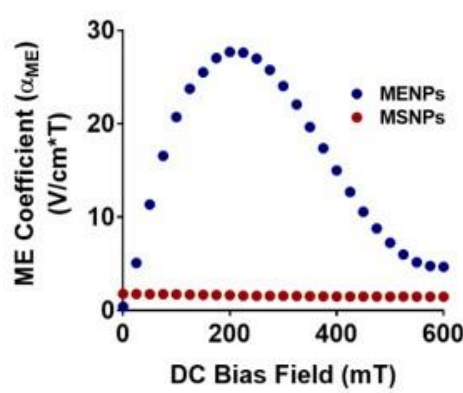

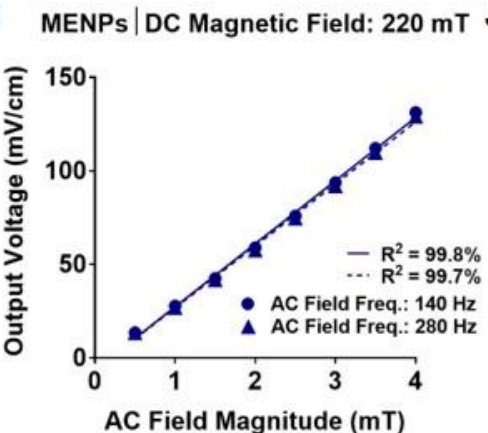

AC Field Magnitude (mT)

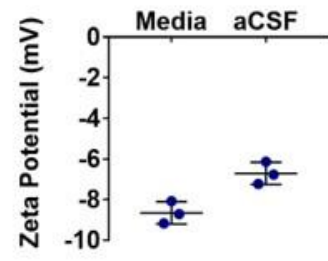

Figure 1. Material and magnetoelectric characterization of MENPs made from magnetostrictive and piezoelectric phases demonstrates wireless electric field generation.

Schematic demonstrating two-phase magnetoelectricity in materials made from magnetostrictive and piezoelectric materials that are strain-coupled (A). Schematic demonstrating the rationale for using a large DC magnetic field overlaid with an AC field to generate optimal magnetoelectric output (B). Diagram of method of in vivo MENP administration. MENPs are injected bilaterally into the thalamic region of mice, and MENPs are wirelessly stimulated using an AC and DC magnetic field (C). Transmission electron microscope (TEM) (D) and TEM-electron energy loss spectroscopy (TEM-EELS) images (E) show MENP morphology and $\mathrm{BaTiO}_{3} / \mathrm{CoFe}_{2} \mathrm{O}_{4}$ phases (green and red, respectively), with quantitative elemental analysis measurement of the molar percentage of each material (E). MENPs were analyzed via X-ray powder diffraction (XRD) to confirm the perovskite crystal structure of $\mathrm{BaTiO}_{3}$ (green) and the spinel crystal structure of $\mathrm{CoFe}_{2} \mathrm{O}_{4}$ (red) (F). Dynamic light scattering (DLS) was used to characterize MENP 
hydrodynamic properties in cell culture media and aCSF $(\mathrm{G})$. The input-output magnetoelectric coefficient was measured as a function of DC bias field in MENPs and MSNPs (H). Output voltage of MENPs was measured using a $220 \mathrm{mT}$ DC field, while varying AC field magnitude (I) or AC field frequency (J). Plots show individual points with mean \pm SD $(n=3)(G)$, and individual points fitted to a linear correlation $(\mathrm{I}, \mathrm{J})$.

Importantly, we found a low correlation $\left(\mathrm{R}^{2}=1.4 \%\right.$ and $1.3 \%$ for $\mathrm{AC}$ magnitude $2 \mathrm{mT}$ and $3 \mathrm{mT}$, respectively) between the output voltage relative to AC field frequency across the range tested $(35-385 \mathrm{~Hz})$, which covers the range of DBS frequencies found to have clinical effect (reviewed in (29)) (Fig. 1J). This frequency range also has little attenuation in tissue, thus improving potential signal penetration depth $(20,21)$.

The effect of wireless MENP signaling on neuronal cell activity was examined in vitro in real-time using intracellular $\mathrm{Ca}^{2+}$ signaling in differentiated human SH-SY5Y cells. MENPs were administered at $100 \mu \mathrm{g} / \mathrm{mL}$ as a suspension in the imaging medium $20 \mathrm{~min}$ prior to testing, using no NPs, MSNPs, and piezoelectric nanoparticles (PENPs) as controls. Prior to choosing a concentration, the toxicity of MENPs was assessed with a lactate dehydrogenase (LDH) assay and a metabolic activity assay (MTS) (Fig. S2). Magnetic stimulation parameters were either no field, a $225 \mathrm{mT}$ (within the maximum $\alpha_{M E}$ range) DC field, a $6 \mathrm{mT}, 140 \mathrm{~Hz}$ AC field, or both DC and AC fields together using a custom coil system (Fig. S3). The DC or AC magnetic fields alone were not expected to output a magnetoelectric effect sufficient to modulate neuronal activity, and were therefore used as controls. We found a significant increase in the percentage of cells exhibiting $\mathrm{Ca}^{2+}$ transients when MENPs were stimulated with a simultaneous AC and DC magnetic field $(20.1 \pm 2.3 \%)$ versus basal activity $(2.8 \pm 2.6 \%)$ (Fig. 2A-C, Movie S1). This increase was not observed when cells were exposed to the AC and DC magnetic stimulation either alone $(1.0 \pm 1.7 \%)$, with MSNPs $(1.4 \pm 1.3 \%)$, or PENPs $(1.4 \pm 1.2 \%)$, which supports our hypothesis that the measured increase in activity was due to magnetoelectric voltage generation. While the MENPs seem to have some effect on neuronal activity $(7.2 \pm 5.0 \%, 5.2 \pm 6.0 \%$, or 3.8 $\pm 5.0 \%$, with no field, AC field only, or DC field only, respectively), this effect was not significantly different than any other negative control group (Fig. B,C; Table S1, Movie S1).

In order to support our hypothesis that the $\mathrm{Ca}^{2+}$ activity we measured was related to electrophysiological cell activity, we stimulated the MENPs with AC and DC magnetic fields, but first treated the cells with either a voltage-gated $\mathrm{Na}^{+}$channel blocker (tetradotoxin, TTX), a voltage-gated $\mathrm{Ca}^{2+}$ channel blocker $\left(\mathrm{Cd}^{2+}\right)$, or an extracellular $\mathrm{Ca}^{2+}$ chelator (ethylene glycolbis( $\beta$-aminoethyl ether)-N,N,N',N'-tetraacetic acid, EGTA) (schematic in Fig. 2D showing drug activity). In the presence of each drug, the cells failed to produce any $\mathrm{Ca}^{2+}$ transients (Fig. 2D,E). This substantiates the dependence of our measured $\mathrm{Ca}^{2+}$ transients on voltage-gated ion channels and extracellular $\mathrm{Ca}^{2+}$ sources, supporting the relationship between our measured $\mathrm{Ca}^{2+}$ activity and cell electrophysiological activity. 

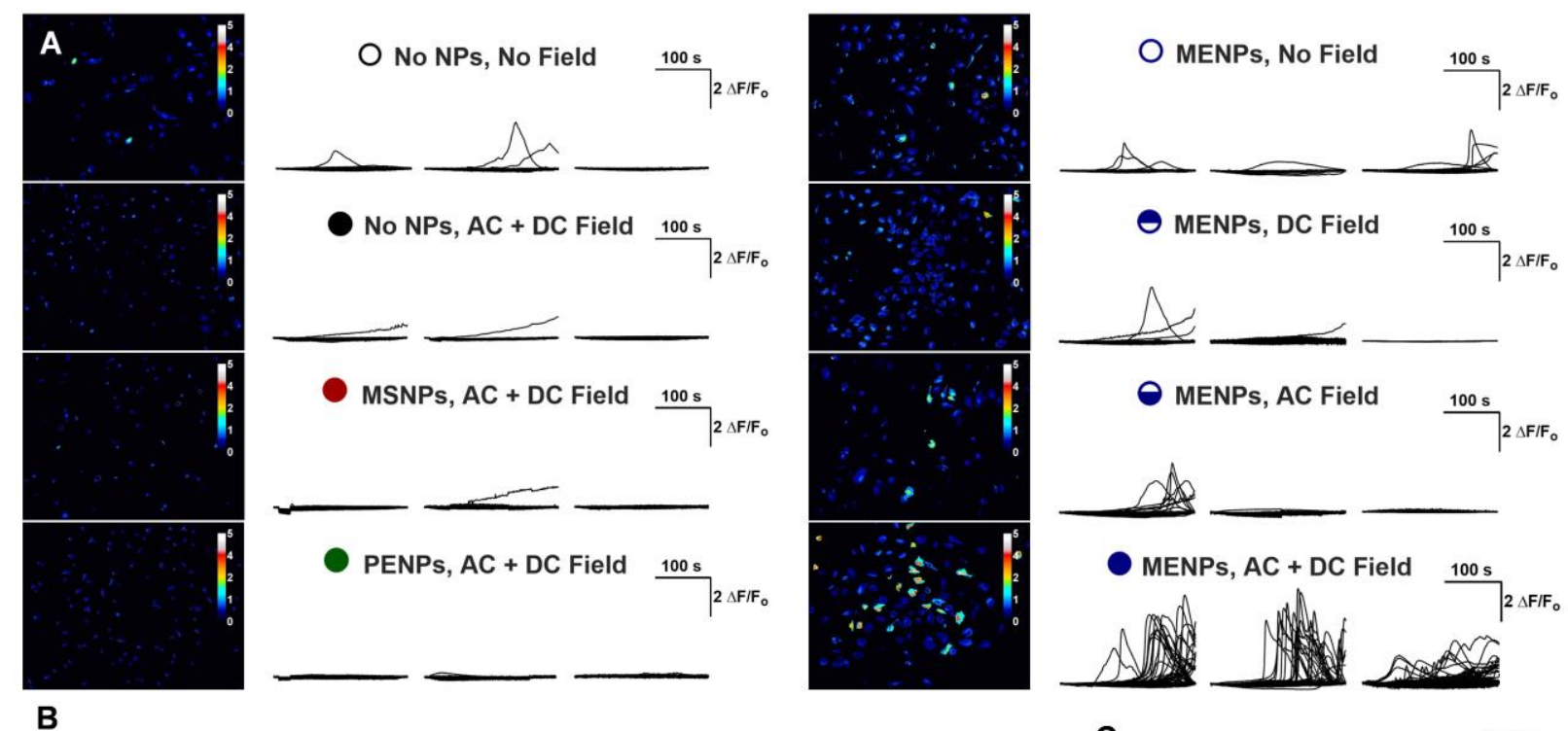

B

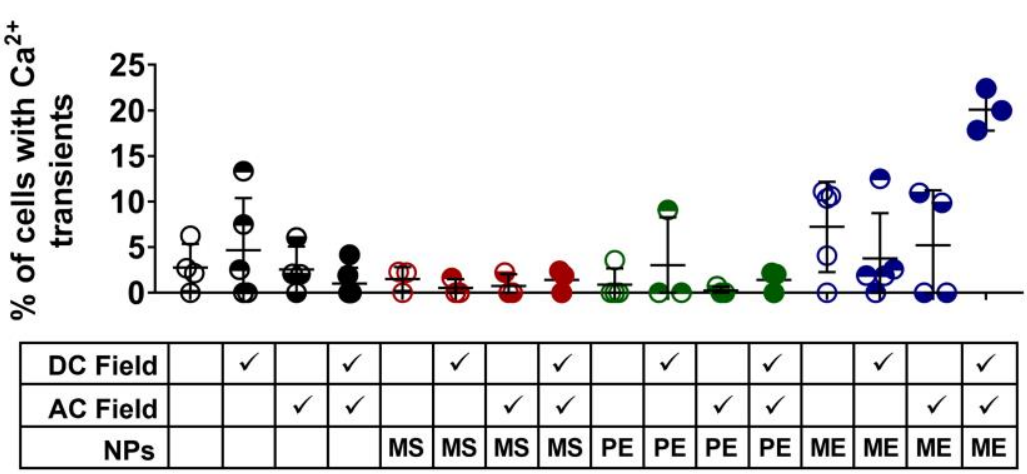

C
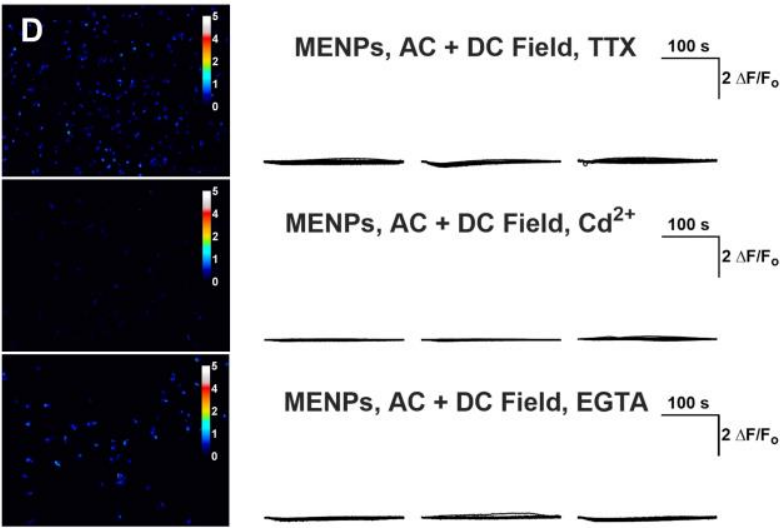

MENPs, AC + DC Field, $\mathrm{Cd}^{2+} \stackrel{100 \mathrm{~s}}{2 \Delta \mathrm{FF} \text { 。 }}$

MENPs, AC + DC Field, EGTA $100 \mathrm{~s}$
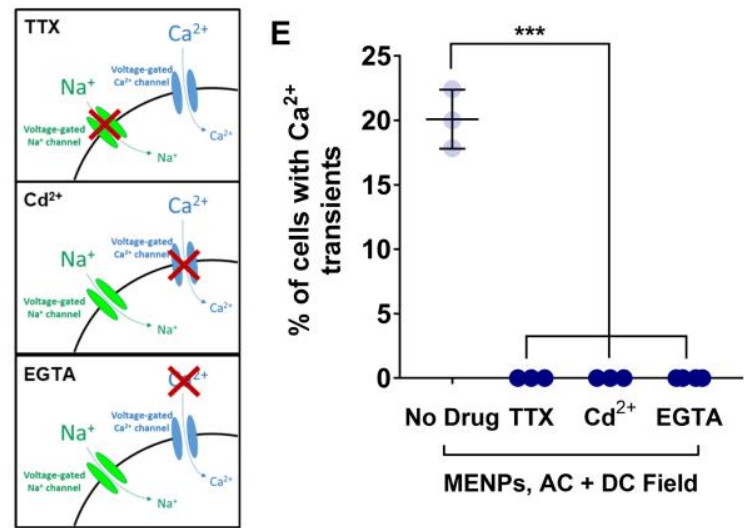

Figure 2. Magnetic stimulation of MENPs modulates neuronal cell activity in vitro. Cells were treated with MENPs, using no NPs, MSNPs, or PENPs as controls, prior to magnetic stimulation. Magnetic stimulation was either $220 \mathrm{mT}$ DC (DC), $6 \mathrm{mT}$ and $140 \mathrm{~Hz}$ AC (AC), or both $\mathrm{DC}$ and $\mathrm{AC}$ fields along the same axis $(\mathrm{AC}+\mathrm{DC})$. Neuronal activity was measured in real time via intracellular $\mathrm{Ca}^{2+}$ imaging using Fluo4 dye, and cell fluorescence was traced over time per cell. Images of total $\mathrm{Ca}^{2+}$ activity over time is shown for selected experimental groups. Calibration bars represent $\Delta \mathrm{F} / \mathrm{F}_{\mathrm{o}}(\mathrm{A})$ The percent of cells demonstrating intracellular $\mathrm{Ca}^{2+}$ transients in (A) is summarized in (B) and significantly different group comparisons are marked in yellow within $(\mathrm{C})$. Cells were treated with TTX, $\mathrm{Cd}^{2+}$, or EGTA prior to treatment with 
MENPs and AC and DC magnetic stimulation, and total $\mathrm{Ca}^{2+}$ activity over time is shown next to a diagram depicting the inhibiting activity of each drug (D). Measured $\mathrm{Ca}^{2+}$ transients of drugtreated cells are summarized with no drug, MENP, AC + DC Field treated cells shown as faded plot points for comparison (E). Plots show traces of $\mathrm{Ca}^{2+}$ activity over time in individual cells $(A, D)$ and individual points with bars showing mean $\pm \mathrm{SD}(\mathrm{B}, \mathrm{E}),(\mathrm{n}=3-6)$; ANOVA with Tukey's post-test (C), or Dunnett's post-test with no drug as the control (E); **p < 0.01, ***p < 0.001, unlabeled group comparisons are not significantly different.

We then sought to assess the feasibility of MENP-based neuromodulation in vivo. MENPs were bilaterally injected into the ventral thalamic region of naïve mice $(\mathrm{C} 57 \mathrm{Bl} / 6 \mathrm{~J})$ at a dose of $100 \mu \mathrm{g} / \mathrm{animal}$, which was found to be tolerable in a dose-toxicity assessment (Fig. S4). This region of the brain was selected as the basal ganglia and thalamus are the most common target areas for DBS (30). Moreover, these areas involve well understood brain circuits in the fields of DBS and neuromodulation for neurological disorders. In addition to the classical basal ganglia model, new models show that parallel circuits also engage associative and limbic regions $(31,32)$. Therefore, the cortico-basal ganglia-thalamo-cortical circuit provides a tool to reliably investigate the effects of neuromodulation on wide range of behavioral functions.

During magnetic stimulation, mice were awake and unrestrained within our in vivo magnetic coil device (Fig. S5). As a control group, mice were treated with MENPs and a DC magnetic field only, meaning they were placed into the magnetic device, but with the AC coil remaining off. We first assessed changes in local neural activity by immunohistochemically measuring the expression of $c$-fos protein, a widely used cell activity marker (33). We found significantly more $c$-fos positive cells in the region of nanoparticle injection when animals were treated with MENPs and an AC and DC field (38.5 \pm 8.0 cells), versus only a DC field (4.25 \pm 3.0 cells) (Fig. 3A-C). This data supports our hypothesis that we could wirelessly modulate local brain activity using the magnetoelectric response of MENPs to magnetic stimulation.

We next wanted to determine if local thalamic neuromodulation induced by MENPs was sufficient to cause modulation in other regions of the cortico-basal ganglia-thalamo-cortical circuit. We found $c$-fos protein expression significantly higher in the motor cortex and nonmotor thalamus following stimulation with MENPs and an AC/DC magnetic field (1046.4 \pm 232.4 , $348.4 \pm 137.7$ cells $/ \mathrm{mm}^{2}$, respectively) versus only a DC magnetic field $(424.8 \pm 214.9,19.9 \pm$ 27.6 cells $/ \mathrm{mm}^{2}$, respectively) (Fig. 3D-G). Importantly, we did not observe a global change in $c$ fos protein expression, such as in the CA1 region of the hippocampus (Fig. 3F,G). Together, these data support our hypothesis that the measured increases in $c$-fos protein expression were due to local thalamic stimulation of the cortico-basal ganglia-thalamo-cortical circuit, and not a nonspecific, global modulation of neural activity via the magnetic field.

To determine if the induced neuromodulation would affect animal behavior, we tested mice via a Rotarod test and an automated CatWalk XT gait analysis system. Mice were injected with MENPs or MSNPs as a control. Behavior with AC and DC magnetic stimulation versus behavior with only DC magnetic stimulation was compared for each mouse. Gait and balancerelated static parameters during the CatWalk test, such as regularity index, run maximum variation, and base of support, showed no significant difference following AC and DC stimulation in either nanoparticle group (MSNPs, $97.3 \pm 2.5$ vs. $97.9 \pm 1.8 \%, 28.3 \pm 7.6$ vs. 31.9 $\pm 11.0 \%, 1.2 \pm 0.1$ vs. $1.2 \pm 0.1 \mathrm{~cm}$, DC vs. AC and DC stimulation, respectively) (MENPs, 97.8 \pm 1.0 vs. $98.1 \pm 1.3 \%, 23.7 \pm 3.3$ vs. $23.6 \pm 8.9 \%, 1.2 \pm 0.1$ vs. $1.2 \pm 0.1 \mathrm{~cm}, \mathrm{DC}$ vs. AC and DC stimulation, respectively) (Fig. 3L-N, Movie S2). Rotarod testing also showed no significant 
difference in latency to fall with either nanoparticle group (MSNPs, $170.4 \pm 72.9$ vs. $170.9 \pm$ $73.2 \mathrm{~s}$; MENPs, $202.6 \pm 40.4$ vs. $184.0 \pm 40.5$ s, DC vs. AC and DC stimulation,) (Fig. 3O). While we anticipated no improvement in motor function, as we tested only naïve mice, these results are important to demonstrate that we saw no detrimental effect to the gait and balance of the animals due to neuromodulation via MENPs. This finding of no generalized behavioral change also corresponds to our $c$-fos expression findings, in which we found only selective expression changes.

Conversely, in analyzing the dynamic parameters of the catwalk test, which are indicative of animal speed, we found a significant difference in the behavior of MENP-treated animals that was not observed in MSNP-treated animals (Fig. 3I-K, Movie S2). The average speed, duty cycle of each limb, and stride length of each limb all changed significantly in MENP-treated mice following AC and DC stimulation $(51.1 \pm 10.9$ vs. $33.6 \pm 4.8 \mathrm{~cm} / \mathrm{s}, 48.1 \pm 3.0 \mathrm{vs} .49 .9 \pm$ $3.5 \%$, and $8.1 \pm 0.5$ vs. $7.3 \pm 0.7 \mathrm{~cm}$, DC vs. AC and DC stimulation, respectively), but not in MSNP-treated mice $(28.3 \pm 5.0$ vs. $29.4 \pm 3.8 \mathrm{~cm} / \mathrm{s}, 51.9 \pm 3.9$ vs. $51.1 \pm 3.2 \%, 6.5 \pm 0.4$ vs. 6.4 $\pm 0.6 \mathrm{~cm}, \mathrm{DC}$ vs. AC and DC stimulation, respectively). As anxiety can be induced via stimulation of the thalamus, we believe that the measured changes in animal speed are due to induced anxiety via selective modulation of the thalamo-cortical circuit. The combined results of $c$-fos protein immunohistochemistry and animal behavioral tests support the conclusion that magnetically-stimulated MENPs wirelessly modulated basal ganglia circuitry to affect brain activity and animal behavior.
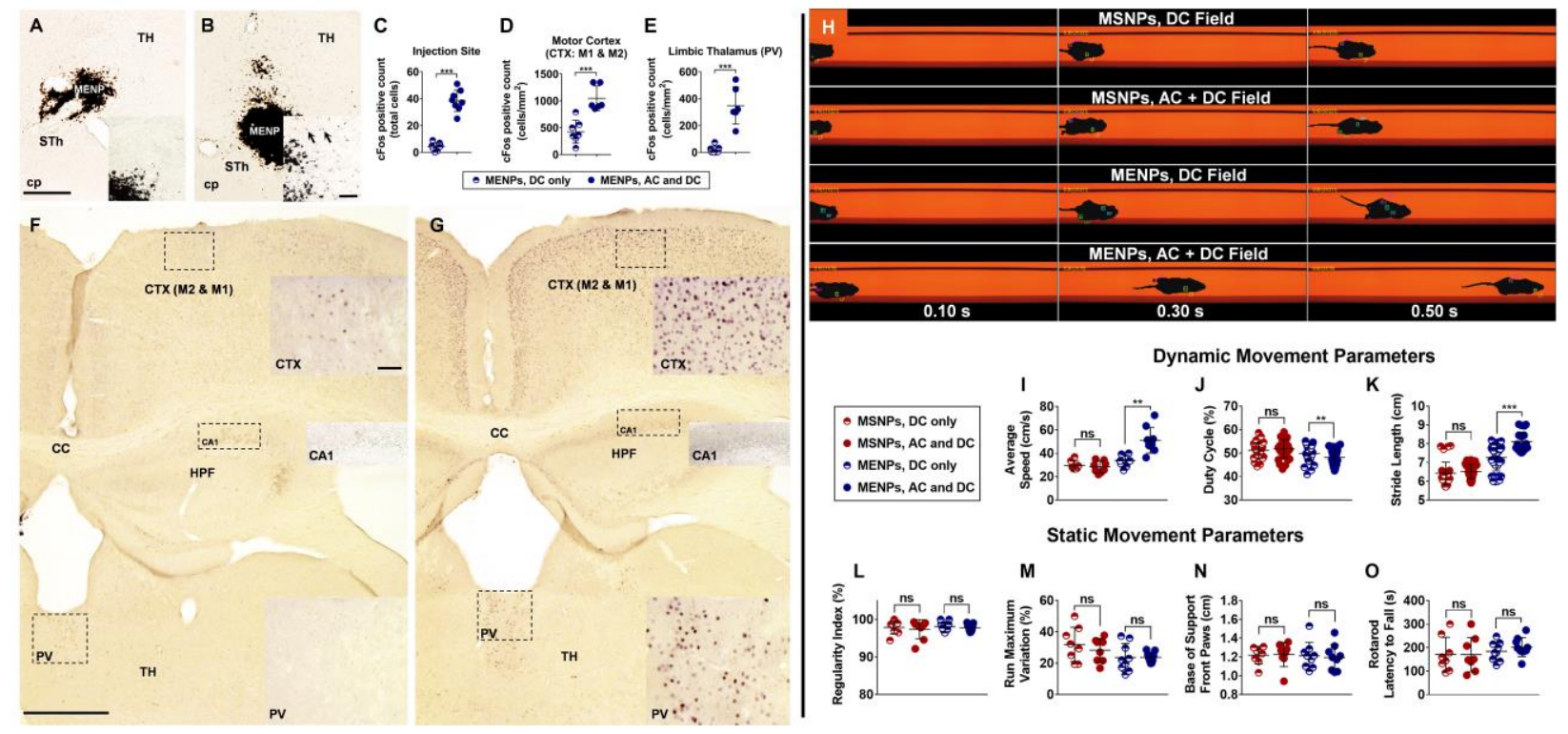

Figure 3. Magnetic stimulation of MENPs locally modulates neural activity in mice, yielding modulation of basal ganglia circuitry and behavioral change. Staining for $c$-fos protein locally to the MENP injection site following DC magnetic stimulation (A) or AC and DC magnetic stimulation (B) shows increased $c$-fos expression in the latter (C). Quantification of $c$ fos expression in the motor cortex (D) and limbic thalamus (E) shows increased expression when MENPs were stimulated with an AC and DC magnetic field $(\mathrm{G})$ versus only a DC magnetic field (F). Time-lapse images showing mouse movement in a catwalk video recording system $(\mathrm{H})$. Dynamic movement parameters as measured by the catwalk recording showed significant 
changes in mouse speed (I), limb duty cycle (J), and limb stride length (K) in MENP-treated mice following AC and DC stimulation versus DC stimulation, while MSNP-treated mice showed no change. Static movement parameters of mouse movement such as regularity index (L), run maximum variation (M), and front-paw base of support $(\mathrm{N})$ as measured by catwalk recording did not significantly change with AC and DC versus DC only magnetic stimulation in either nanoparticle group. Rotarod latency to fall also did not significantly change with AC and DC versus DC only magnetic stimulation in either nanoparticle group (O). Scale bar, $250 \mu \mathrm{m}$ (overview) and $50 \mu \mathrm{m}$ (inset) (A, B, F, G). Plots show individual points with bars showing mean $\pm \mathrm{SD}(\mathrm{C}-\mathrm{E}, \mathrm{I}-\mathrm{O}),(\mathrm{n}=6-9$ mice, individual limb values for $\mathrm{J}, \mathrm{K})$; Unpaired t-test $(\mathrm{C}-\mathrm{E})$, or paired $\mathrm{t}$-test $(\mathrm{I}-\mathrm{O}), \mathrm{ns}=$ not significant, $* * \mathrm{p}<0.01, * * * \mathrm{p}<0.001$.

\section{Conclusion}

This work demonstrates the potential of magnetoelectric materials as nanoelectrodes for wireless electrical modulation of deep brain targets. Herein, we have shown that we can stimulate MENPs with a magnetic field to remotely generate electric polarization of the MENPs. We have shown evidence that non-resonant frequency magnetic stimulation of MENPs locally modulates neuronal activity in vitro and in vivo. We have also demonstrated that this modulation is sufficient to change animal behavior, and to modulate other regions of the cortico-basal ganglia-thalamo-cortical circuit. Future work will be key to optimizing magnetoelectricity based neural devices and understanding the abilities and limitations of this technology. Magnetoelectric nanoelectrodes show promise for new technologies in wireless neural devices. 


\section{Materials and Methods}

\section{$\underline{\text { Magnetoelectric nanoparticle (MENP) synthesis }}$}

MENPs were synthesized in a manner similar to Corral-Flores et al. (29). $\mathrm{CoFe}_{2} \mathrm{O}_{4}$ nanoparticles (30 nm, Sigma-Aldrich) were suspended in $\mathrm{dH}_{2} \mathrm{O}$ at a concentration of $10 \mathrm{mg} / \mathrm{mL}$ to $80^{\circ} \mathrm{C}$ while stirring. Oleic acid was added to the suspension at $30 \mathrm{wt} . \%$ with respect to $\mathrm{CoFe}_{2} \mathrm{O}_{4}$, the temperature was raised to $90^{\circ} \mathrm{C}$ for $30 \mathrm{~min}$, then lowered to $60^{\circ} \mathrm{C}$. Octane was added to the suspension at a 1:1 ratio to the $\mathrm{dH}_{2} \mathrm{O}$ volume, which separated oleic acid coated $\mathrm{CoFe}_{2} \mathrm{O}_{4}$ particles into the organic layer. The organic layer was then washed with $\mathrm{dH}_{2} \mathrm{O}$ three times. Barium acetate (BaAc) and titanium butoxide (TiButO) were dissolved in glacial acetic acid with stearic acid (final concentration $0.01 \%$ ) such that the final molar ratio of $\mathrm{BaTiO}_{3}$ to $\mathrm{CoFe}_{2} \mathrm{O}_{4}$ was 1:3. This solution was stirred and heated to $90^{\circ} \mathrm{C}$, the $\mathrm{CoFe}_{2} \mathrm{O}_{4}$ solution was added, as well as 2-methoxyethanol at a final volume concentration of $30 \%$. The solution was dried, calcined at $700^{\circ} \mathrm{C}$ for $2 \mathrm{~h}$, and then ground with a mortar and pestle. To select for particles with better colloidal stability, MENPs were suspended in $\mathrm{dH}_{2} \mathrm{O}$, centrifuged for $1 \mathrm{~min}$ at $10 \mathrm{G}$, and particles within the supernatant were kept for further experiments.

\section{$\underline{\text { X-ray diffraction (XRD) analysis of MENP crystal structure }}$}

XRD analysis of MENPs was carried out on a Bruker D8 Advance Powder Diffractometer using $\mathrm{Cu}$ radiation generated at $40 \mathrm{kV} / 40 \mathrm{~mA}$ with a Bragg-Brentano beam path. A divergence slit at $0.5^{\circ}$, anti-scatter slits at $2^{\circ}$ and $4^{\circ}$, and Soller slits were used. The output beams were received using a VÅNTEC-1 1-dimensional detector. Peaks were identified using the International Centre for Diffraction Data (ICDD) database. MathWorks® MATLAB software was used to baseline-correct the spectrum, using the msbackadj function.

Elemental Analysis of MENPs to determine chemical composition

MENP elemental analysis was carried out via Inductively Coupled Plasma - Optical Emission Spectrometry (ICP-OES) using a Spectro Ciros spectrometer (Kleve, Germany). MENPs were first dissolved in an aqueous solution of $3 \% \mathrm{HNO}_{3}$ and $1 \% \mathrm{HF}$ prior to sample loading in the spectrometer. Data were analyzed using Spectro ICP Analyzer software to detect $\mathrm{Ba}, \mathrm{Ti}, \mathrm{Co}$ and Fe spectra. Data are presented as the mean $\pm \mathrm{SD}$ of each element measured within $\mathrm{BaTiO}_{3}$ and $\mathrm{CoFe}_{2} \mathrm{O}_{4}$.

Transmission electron microscopy (TEM) and electron energy loss spectroscopy (EELS) analysis of MENP morphology

MENPs were prepared for TEM analysis by drop-casting an aqueous suspension onto Ccoated-Cu TEM grids and air-drying. TEM and TEM-EELS images were acquired using a ZEISS Sub-Electron-volt Sub-Angstrom Microscope (SESAM). Data was acquired in TEM mode at $200 \mathrm{kV}$. For EELS, we acquired energy-filtered TEM (EFTEM) spectrum images from 30 to $120 \mathrm{eV}$, with $3 \mathrm{eV}$ steps and $4 \mathrm{X}$ binning. After data acquisition, the EELS signal from Ba $\left(\mathrm{N}_{4,5}\right.$ edge, $\left.90 \mathrm{eV}\right)$ and $\mathrm{Fe}\left(\mathrm{M}_{2,3}\right.$ edge, $\left.54 \mathrm{eV}\right)$ was extracted and used for the elemental map.

\section{Analysis of MENP hydrodynamic properties}

Hydrodynamic diameter and zeta-potential of MENPs were measured via dynamic light scattering (DLS) using a Wyatt Mobius ${ }^{\text {TM }}$ DLS Instrument and analyzed via Wyatt DYNAMICS 
software. MENPs were diluted to a concentration of $100 \mu \mathrm{g} / \mathrm{mL}$ in either our cell culture differentiation media (see below) or an artificial cerebrospinal fluid (aCSF) solution (34) during the measurements. Data was analyzed from three independent experiments.

Formation of sintered pellets of nanoparticles and pellet wiring

For ME measurement of pellets, $0.65 \mathrm{~g}$ of MENPs were mechanically pressed into a pellet of diameter $8 \mathrm{~mm}$ using 6 tonnes $/ \mathrm{cm}^{2}$ of pressure, then sintered at $1150^{\circ} \mathrm{C}$ for $12 \mathrm{~h}$. MSNP pellets were prepared in the same way but using only $\mathrm{CoFe}_{2} \mathrm{O}_{4}$ nanoparticles. The circular surfaces of the pellets were painted with conductive silver glue to attach copper plates (Fig. S1A). Pellets were heated to $140^{\circ} \mathrm{C}$, electrically poled at $1 \mathrm{kV} / \mathrm{mm}$ thickness for $5 \mathrm{~min}$, then allowed to cool to room temperature while maintaining the applied voltage. The pellets were then wired to a charge amplifier. The pellet and charge amplifier were enclosed in a Faraday shield, and connected externally to a lock-in amplifier for voltage measurement (Fig. S1B-H).

\section{Design of charge amplifier}

For electrical measurement of the magnetoelectric response of pellets, a charge amplifier is used to eliminate the effects of stray capacitance on the measurement of the piezoelectric charge. The battery-powered amplifier was constructed on a standard FR4 printed circuit board, which was placed within the Faraday. The charge amplifier uses an operational amplifier circuit (Fig. S1F-H) based on the Texas Instruments OPA340. The amplifier has a high-pass characteristic with a $-3 \mathrm{~dB}$ frequency of $3 \mathrm{~Hz}$, and the calculated gain of the circuit in the passband is $200 \mathrm{mV} / \mathrm{pC}$.

\section{$\underline{\text { Magnetoelectricity measurements }}$}

A Microsense EZ vibrating sample magnetometer (VSM) was used as a DC magnetic field source, and was modified to hold an additional, smaller Helmholtz coil. This was powered with a signal generator $(35-385 \mathrm{~Hz}$ sine wave) connected to a linear voltage amplifier (Hewlett Packard) to provide current to the smaller coils, generating an AC magnetic field in the plane of the sample. The pellet was oriented such that the AC and DC magnetic fields were parallel to the pellet's central axis (Fig. S1E). The AC magnetic field magnitude was measured using a gaussmeter prior to experimentation. Pellets were demagnetized prior to all measurements.

\section{Culture and differentiation of SH-SY5Y neuronal cells}

SH-SY5Y cells were purchased from DSMZ (ATCC@ CRL-2266 ${ }^{\mathrm{TM}}$ ). Maintenance cultures were grown in DMEM/F12 (Gibco) with $10 \%$ fetal bovine serum (FBS) and $1 \%$ penicillin/streptomycin, at $37^{\circ} \mathrm{C}$ with $5.0 \% \mathrm{CO}_{2}$. Media was changed every 3 to 4 days. Prior to plating for experiments, wells were coated with $5 \mu \mathrm{g} / \mathrm{mL}$ laminin in phosphate-buffered saline (PBS) with $\mathrm{Ca}^{2+} / \mathrm{Mg}^{2+}$ for $1 \mathrm{~h}$ at $37^{\circ} \mathrm{C}$. For $\mathrm{Ca}^{2+}$ signaling experiments, cells were plated at a concentration of $20,000 / \mathrm{cm}^{2}$ onto cell culture treated, 4-well IBIDI ${ }^{\circ} \mu$-slides. For toxicity analysis, cells were plated at a concentration of $20,000 / \mathrm{cm}^{2}$ onto cell culture treated 96 -well plates. Experimental cultures were differentiated in DMEM/F12 medium containing 1\% FBS, $1 \%$ penicillin/streptomycin, and $10 \mu \mathrm{M}$ retinoic acid (Sigma-Aldrich) for 4 days prior to all experiments. 


\section{Analysis of cell toxicity}

MENPs were suspended in experimental cell culture medium at a concentration of 0,50 , 100, 200, or $300 \mu \mathrm{g} / \mathrm{mL}$ and added to cells. Toxicity was assessed at $24 \mathrm{~h}$ following MENP administration via a CyQUANT ${ }^{\mathrm{TM}}$ lactate dehydrogenase (LDH) assay kit (ThermoFisher Scientific), as well as a CellTiter 96® AQueous One MTS assay. Assay results were read using a BioTek ${ }^{\circledR}$ Synergy ${ }^{\mathrm{TM}} 2$ Microplate Reader (Fig. S2). Each experiment was tested within 4 wells, and the average of these values was recorded to provide a single data point. The data was analyzed from three independent experiments.

\section{$\underline{\text { In vitro magnetic stimulation }}$}

A magnetic stimulation setup was designed to fit into a Zeiss Axio Observer A1 microscope, and to hold a 4-well IBIDI® $\mu$-slide (Fig. S3). A DC magnetic field was provided by three permanent $\mathrm{NdFeB}$ magnets (N42, $6 \mathrm{~cm}$ diameter, $5 \mathrm{~mm}$ height; Supermagnete) on either side of the cells to generate a $225 \mathrm{mT}$ field at the center of the cell culture well. A magnetic coil was used to provide an AC magnetic field along the same axis. AC signals were generated by a National Instruments ${ }^{\mathrm{TM}}$ DAQ USB X-Series device, controlled via LabVIEW software, and amplified by a class D audio amplifier. For all experiments with AC magnetic stimulation, the AC field component was a $6 \mathrm{mT}$ sine wave at $140 \mathrm{~Hz}$ applied during the time window of $10-30$ $\mathrm{s}$ during the time lapse recording. AC and DC magnetic field magnitudes were verified with a magnetometer.

\section{$\underline{\mathrm{Ca}^{2+} \text { signaling experiments }}$}

Cells were loaded with $1 \mu$ M Fluo4-AM dye (ThermoFisher Scientific) in Live Cell Imaging Solution (LCIS, Invitrogen) for $30 \mathrm{~min}$ at $37^{\circ} \mathrm{C}$. Experimental suspensions of no NPs, MENPs, PENPs, or MSNPs were prepared at $100 \mu \mathrm{g} / \mathrm{mL}$ in LCIS. After Fluo4 loading, cells were washed $3 \mathrm{X}$ with LCIS, and particle suspension solutions were added. Cells with particles were incubated for $20 \mathrm{~min}$ at $37^{\circ} \mathrm{C}$ to allow Fluo4 to de-esterify, then moved onto a Zeiss Axio Observer A1 microscope mounted with the in vitro coil system. For experiments using inhibitory drugs, Fluo4 loading was carried out as described above, and drugs were added in the LCIS solution with MENPs after washing. For EGTA, PBS was used instead of LCIS, and was added during the Fluo4 loading step. TTX was added at a concentration of $100 \mathrm{nM}, \mathrm{CdCl}_{2}\left(\mathrm{Cd}^{2+}\right)$ was added at $100 \mu \mathrm{M}$, and EGTA at $5 \mathrm{mM}$, which have previously been determined to be inhibitory but nontoxic concentrations (14).

Fluo4 was excited using a $470 \mathrm{~nm}$ LED with a 484/25 nm excitation filter, and observed through a 519/30 nm emission filter. Time lapse images were taken at 10X magnification, every $1 \mathrm{~s}$ for $240 \mathrm{~s}$ using $50 \mathrm{~ms}$ illumination, and recorded using a Zeiss Axiocam 503 mono camera (2.8 megapixels). Data was collected from $3-6$ independent experiments per group.

Time lapse recordings were analyzed using ImageJ software. Briefly, the first 10 images of each time lapse were stacked into a single image to enable region of interest (ROI) selection. Following brightness normalization, blurring, background subtraction, and thresholding, ROIs were selected from this image using the Analyze Particles function (with all settings remaining consistent for all time lapses). These ROIs were then overlaid onto the completely unmodified time lapse series, and the mean gray value within each ROI was recorded for each frame. These values were then used to calculate $\mathrm{Ca}^{2+}$ transient amplitudes as $\Delta \mathrm{F} / \mathrm{F}_{\mathrm{o}}$. Cells positively showing $\mathrm{Ca}^{2+}$ transients were calculated using MathWorks ${ }^{\circledR}$ MATLAB software, using a linear baseline 
correction and the peakfinder function. Images in Fig. 2A,D were generated by creating a maximum value Z-stack of the entire video.

\section{Animals}

Experiments were performed on 68 male naïve mice (C57Bl/6J; Jackson Laboratory). Mice were socially housed under controlled conditions $\left(21 \pm 2^{\circ} \mathrm{C}\right.$ and $40-60 \%$ humidity) in a reversed 12h day/night cycle (lights on, 7 p.m.) until they had received surgery. Mice were given ad libitum access to food and water. At the time of surgery, mice were 3 months of age.

Experiments were conducted according to the directive 2010/63/EU for animal experiments and in agreement with the Animal Experiments and Ethics Committee of the Maastricht University, Maastricht, The Netherlands.

\section{$\underline{\text { Stereotactic nanoparticle administration }}$}

Buprenorphine $0.1 \mathrm{mg} / \mathrm{kg}$ was subcutaneously injected half an hour prior to surgery as an analgesic. Inhalational anesthesia was induced and maintained with isoflurane (Abbot Laboratories, Maidenhead, UK) at 4\% and 1.5-3\%, respectively. After adequate induction of the anesthesia, the mouse was placed in a small animal stereotaxic frame (Stoelting, Dublin, Ireland) and fixed by ear bars with zygoma ear cups (Kopf, Los Angeles, United States of America) and a mouse gas anesthesia head holder (Stoelting, Dublin, Ireland). To maintain body temperature at $37^{\circ} \mathrm{C}$ throughout the whole procedure, the mouse was placed on a thermo-regulator pad. An ocular lubricant was applied to prevent drying of the eyes. A subcutaneous injection of Lidocaine $1 \%$ (Streuli Pharma, Uznach, Switzerland) at the incision side was given for local anesthesia.

Consecutively, burr holes above the subthalamic area (AP: $-2.06 \mathrm{~mm}$, ML: $-1.50 \mathrm{~mm}$, DV: -4.50) was made and a total of $2 \mu 1$ of MENPs or MSNPs were injected with a microinjection apparatus Nanoject II (Drummond Scientific). In phase-I in vivo experiment MENPs injection was conducted only in right hemisphere to compare microglia and astrocytes population between injected and intact hemispheres. The infusion rate was $100 \mathrm{nl} / \mathrm{min}$. After the injections, the syringe needle remained inside the brain for another $10 \mathrm{~min}$ prior to a slow withdrawal.

\section{In vivo magnetic stimulation}

All in vivo magnetic stimulation was carried out using a custom coil system that would allow mice to move freely during the experiments. The animal experiment setup was designed to provide a $220 \mathrm{mT}$ DC magnetic field with a $6 \mathrm{mT}, 140 \mathrm{~Hz}$ AC magnetic field along the same axis at the center of the animal chamber. Images and the design of the in vivo coil system are shown in Figure S5. The structure was 3D-printed with Acrylonitrile Butadiene Styrene (ABS) using a uPrint SE Plus 3D printer. A DC magnetic field was provided by six NdFeB disk magnets (N42, $6 \mathrm{~cm}$ diameter, $5 \mathrm{~mm}$ height; Supermagnete) on each side of the animal chamber. As safety precautions, the permanent magnets were covered with a protective lid, and the animal holder base was 3D-printed using the solid option for higher durability. The AC magnetic field was provided by two coils on either side of the animal chamber. A $1 \mathrm{~mm}$ thick copper wire was wound around a 3D-printed plastic coil frame with 360 turns each. Corresponding coil-pair resistance was $4.94 \mathrm{Ohm}$, and coil-pair inductance was $24.5 \mathrm{mH}$. A Voltcraft 8210 signal generator was used to provide a $140 \mathrm{~Hz}$ sine wave, which was amplified using a QSC-GX7 power amplifier. These were then connected to the AC coils. AC and DC magnetic field magnitudes were verified with a magnetometer. For all AC and DC stimulation experiments, 
mice were stimulated with the coil turned on for 120 s. For DC only stimulation experiments, mice were placed into the animal chamber for $120 \mathrm{~s}$ with the coil remaining off.

\section{Description and timelines of animal experimental procedures}

\section{Phase I: Toxicity assessment}

We first adjusted optimal concentration of MENPs. Three doses were tested, including; 25, 50 and $100 \mathrm{mg} / \mathrm{ml}$. Mice were randomly assigned to either: 25,50 or $100 \mathrm{mg} / \mathrm{ml}$ test groups $(\mathrm{n}=$ 8) and received stereotactic injection of MENPs (Fig. S4A). Animals were monitored for signs of sub- or epidural hemorrhage, neurological symptoms of the injection, welfare (weight, responsiveness, water intake), discomfort/pain. No animals were eliminated from the experiments due to failing these criteria. Fourteen days after the surgery, mice were sacrificed for immunohistochemical (IHC) analysis of the brain as described below. Brain sections were processed using antibodies raised against astrocytes and microglia (Fig. S4B,C). Another series of brain sections were stained using standard hematoxylin and eosin (H\&E) to evaluate tissue damage at the site of injection (Fig. S4D).

\section{Phase II: Persistence of nanoparticles at injection site and c-fos protein expression}

Mice were randomly assigned to three test groups $(n=8)$ and received stereotactic injection of MENPs $(100 \mathrm{mg} / \mathrm{ml})$. We tested the washout of MENPs at different time-points including 48 hours, 2 and 4 weeks (Fig. S4E,F). At the end of each time-pint, mice underwent transcardial perfusion and brains were removed and used for IHC and H\&E analysis. In order to evaluate $c$ fos protein expression, two hours prior to perfusion, half of the mice in each group underwent magnetic stimulation for $120 \mathrm{~s}$. As a control group, the other half of the mice were placed in the coil with no current running through the coil, exposing them only to the DC magnetic field of the permanent magnets.

\section{Phase III: Behavioral testing}

In order to evaluate the effect of MENP-induced neuronal modulation on brain tissue, two groups of animals were tested and behavioral responses were evaluated. Mice were randomly assigned into two groups $(\mathrm{n}=10)$ and received stereotactic injection of either MENPs or MSNPs $(100 \mathrm{mg} / \mathrm{ml})$. Following the recovery period of 1 week post-surgery, animals were stimulated in the magnetic field and behavioral testing was conducted. Specifically, animals were stimulated with either an AC and DC magnetic field (in the in vivo coil system with the coil on), or with only a DC magnetic field (in the in vivo coil system with the coil off). Measured behavioral parameters were compared between the same mice following stimulation with an AC and DC magnetic field versus stimulation with only a DC magnetic field. At the end of the behavioral testing phase ( 6 weeks post-surgery), mice underwent transcardial perfusion as described below, and brains were removed and used for IHC analysis (Fig. S4G).

\section{Behavioral testing}

\section{CatWalk video recording}

An automated gait analysis system CatWalk XT (Noldus 7.1, Wageningen, the Netherlands) was to evaluate motor behaviour. The CatWalk consists of an enclosed walkway with a glass plate and a speed video recording camera (Fig. 3H). Gait performance was assessed and recorded using the CatWalk analysis software. The glass plate was cleaned and dried before testing each subject to minimize the transmission of olfactory clues and prevent animals from 
stopping to smell or explore something during a run. In general, one successful test recording consisted of an average of five uninterrupted runs having a comparable running speed with a maximum variation of $30 \%$. The following 20 static and dynamic parameters assessing individual paw functioning and gait patterns were analyzed: stance, mean intensity, print area, print length, print width, swing mean, swing speed, stride length, maximum intensity at maximum contact, maximum intensity, minimum intensity, step cycle, duty cycle, regularity index, base of support of the forelimbs, base of support of the hindlimbs, three limb support, speed, and cadence.

\section{Rotarod test}

An accelerating rotarod with a grooved rotating beam $(3 \mathrm{~cm})$ raised $16 \mathrm{~cm}$ above a platform (model 47650, Ugo Basile Biological Research Apparatus, Italy) was used to measure coordination. The latency to fall off the rotating rod was recorded. Data were expressed as the mean value from three trials. Mice were subjected to four $300 \mathrm{~s}$ trials per day for three consecutive days (days 1-3) with an inter-trial interval of $\sim 15 \mathrm{~min}$. Mice were forced to run on a rotating drum with speeds starting at $4 \mathrm{rpm}$ and accelerating to $40 \mathrm{rpm}$ within $300 \mathrm{~s}$. Mice remaining on the beam during the full $300 \mathrm{~s}$ of the task were taken from the rotarod and given the maximum score. Animal sacrifice protocol for immunohistochemical analysis of brain tissue

Mice were deeply anaesthetized with pentobarbital and transcardially perfused with tyrode buffer, followed by ice-cold $4 \%$ paraformaldehyde fixative in $0.1 \mathrm{M}$ phosphate buffer. The brains were extracted from the crania and post-fixed in $4 \%$ paraformaldehyde overnight, then submerged in sucrose for cryoprotection ( 24 hours in $20 \%$ sucrose at $5^{\circ} \mathrm{C}$ ). Coronal brain sections $(20 \mu \mathrm{m})$ were cut on a cryostat and stored at $-80^{\circ} \mathrm{C}$.

\section{Immunohistochemistry}

Tissue sections were incubated overnight with polyclonal rabbit antibodies raised against c-fos protein (1:1000; Santa Cruz Biotechnology Inc.; sc-253), GFAP (1:1000; Dako; Z-033429), or Iba-1(1:1000; Wako; 016-26461). c-fos IHC used biotinylated donkey anti-rabbit secondary antibody (1:400; Jackson Immunoresearch Laboratories Inc.; 711065152) and avidin-biotin peroxidase complex (1:800, Elite ABC-kit, Vectorlabs; PK-6100). The staining was visualized by 3,3'-Diaminobenzidine (DAB) combined with $\mathrm{NiCl} 2$ intensification. GFAP and Iba-1 were visualized using immunofluorescence with donkey anti-rabbit Alexa 488 (1:100; Invitrogen; A21206). Due to suboptimal perfusion fixation in some animals, brains were not processed for IHC.

Quantification of $c$-fos immunohistochemically labeled cells:

Photographs of the stained motor cortex and thalamus sections from 3 rostrocaudal anatomical levels from bregma (AP -0.58, -0.94 and -1.22) were taken at $10 \mathrm{X}$ magnification. We used Cell P software (Olympus Soft Imaging Solutions, Münster, Germany) from an Olympus U-CMAD-2 digital camera connected to an Olympus AX 70 microscope (Olympus, Zoeterwoude, The Netherlands). In the images of the area of interest, the number of c-Fospositive cells were counted using ImageJ software (version 1.52; NIH, Bethesda, USA). Cells immunopositive for $c$-fos were counted manually, and the mean number of cells was corrected for surface area and expressed as cells $/ \mathrm{mm}^{2}$. A cell was regarded positive when the intensity of 
the cell staining was significantly higher than the surrounding background. The average value of three sections was used for statistical analysis in each subject. For the subthalamic nucleus, a digital photograph was taken at one anatomical bregma (-2.06) and all $c$-fos positive cells within $1 \mathrm{~mm}^{2}$ of the injection site were counted.

Quantification of GFAP and Iba-1 immunohistochemically labeled cells:

Photographs of the stained motor cortex and thalamus sections from 3 rostrocaudal anatomical levels from bregma (AP -1.70, -2.06 and -2.30) were taken at 10x magnification. We used Cell P software (Olympus Soft Imaging Solutions, Münster, Germany) from an Olympus U-CMAD-2 digital camera connected to an Olympus AX 70 microscope (Olympus, Zoeterwoude, The Netherlands). In the images of the area of interest, fluorescent density was measured using ImageJ software (version 1.52; NIH, Bethesda, USA). The average value of three sections was used for statistical analysis in each subject.

\section{$\underline{\text { Statistical analysis }}$}

Unless otherwise indicated, data are presented as individual values with bars showing the mean \pm standard deviation. The AC magnetic field magnitude and frequency dependence on MENP voltage output was determined using a linear regression, with coefficient of determination presented as $\mathrm{R}^{2}$. In vitro $\mathrm{Ca}^{2+}$ transient activity and in vivo cFos expression were analyzed using a one-way analysis of variance (ANOVA) with Tukey's post-test to compare all groups (Fig. S4). In vitro analysis of $\mathrm{Ca}^{2+}$ signaling with inhibitors was analyzed using a oneway ANOVA with Dunnett's post-test, using drug-untreated cells as the controls. $c$-fos protein expression in brain tissue was analyzed using an unpaired t-test. Changes in behavioral parameters in the same mice following stimulation with either a DC magnetic field or an AC and DC magnetic field were analyzed using a paired t-test. $\mathrm{p}$-values $<0.05$ were considered statistically significant in all cases. 
Acknowledgments: The authors would like to acknowledge Mr. Kerstin Hahn and Prof. Peter van Aken of the Stuttgart Center for Electron Microscopy (StEM, Max Planck Insitute for Solid State Research) for their work capturing TEM images of nanoparticles. We would also like to thank Dr. Samir Hammoud of the Chemical Synthesis facility (Max Planck Insitute for Intelligent Systems) for completing elemental analysis of our nanoparticles. We would also like to thank Mr. Gerd Maier and Dr. Gunther Richter of the Materials Central Scientific Facility (Max Planck Institute for Intelligent Systems) for carrying out XRD analysis of our nanoparticles. Funding: KK thanks the Institute for International Education and the Whitaker International Program for fellowship support. AJ thanks the Nederlandse Organisatie voor Wetenschappelijk Onderzoek (NWO). This work was funded by the Max Planck Society. HG thanks the Alexander von Humboldt Foundation for fellowship support. Author contributions: $\mathrm{KK}, \mathrm{AJ}, \mathrm{MS}$, and YT conceived the project. KK and AJ designed the experimental layout, analyzed all data, and supervised experiments. KK wrote the manuscript, with the assistance of AJ. YY designed the in vitro data acquisition method. KK, YY, and DF carried out in vitro modulation experiments. HG conceived and carried out magnetoelectricity measurements. OE and $\mathrm{HG}$ designed and fabricated the in vitro coil system. OE designed and fabricated the in vivo coil system. AJ completed all in vivo data acquisition, with the assistance of FA. All authors reviewed and edited the manuscript. Competing interests: The authors have no competing interests. Data and materials availability: All data is available in the main text or the supplementary materials.

\section{Supplementary Materials:}

Figures S1-S5

Table S1

Movies S1-S2

\section{References and Notes:}

1. A. L. Benabid, P. Pollak, A. Louveau, S. Henry, J. de Rougemont, Combined (thalamotomy and stimulation) stereotactic surgery of the VIM thalamic nucleus for bilateral Parkinson disease. Appl Neurophysiol 50, 344-346 (1987).

2. F. B. Wagner et al., Targeted neurotechnology restores walking in humans with spinal cord injury. Nature 563, 65-71 (2018).

3. J. Rivnay, H. Wang, L. Fenno, K. Deisseroth, G. G. Malliaras, Next-generation probes, particles, and proteins for neural interfacing. Science Advances 3, e1601649 (2017).

4. E. de Schlichting et al., Pulse generator battery life in deep brain stimulation: out with the old... in with the less durable? Acta Neurochir (Wien) 161, 2043-2046 (2019).

5. P. Yeon, M. S. Bakir, M. Ghovanloo, in 2018 IEEE Custom Integrated Circuits Conference (CICC), 2018. pp. 1-4.

6. S. Lee et al., A $250 \mu \mathrm{m} \times 57 \mu \mathrm{m}$ Microscale Opto-electronically Transduced Electrodes (MOTEs) for Neural Recording. IEEE transactions on biomedical circuits and systems 12, 1256-1266 (2018).

7. S. Lee et al., in 2018 IEEE International Solid-State Circuits Conference-(ISSCC), 2018. pp. 292-294. 
8. Y. K. Song et al., Active microelectronic neurosensor arrays for implantable brain communication interfaces. IEEE Trans Neural Syst Rehabil Eng 17, 339-345 (2009).

9. D. Seo et al., Wireless recording in the peripheral nervous system with ultrasonic neural dust. Neuron 91, 529-539 (2016).

10. M. M. Ghanbari et al., A Sub- $\mathrm{mm}^{3}$ Ultrasonic Free-Floating Implant for Multi-Mote Neural Recording. IEEE Journal of Solid-State Circuits 54, 3017-3030 (2019).

11. D. Seo, J. M. Carmena, J. M. Rabaey, M. M. Maharbiz, E. Alon, Model validation of untethered, ultrasonic neural dust motes for cortical recording. J Neurosci Methods 244, 114-122 (2015).

12. B. C. Johnson et al., in 2018 IEEE Custom Integrated Circuits Conference (CICC), 2018. pp. 1-4.

13. D. K. Piech et al., A wireless millimetre-scale implantable neural stimulator with ultrasonically powered bidirectional communication. Nature Biomedical Engineering 4, 207-222 (2020).

14. A. Marino et al., Piezoelectric Nanoparticle-Assisted Wireless Neuronal Stimulation. ACS Nano 9, 7678-7689 (2015).

15. R. Chen, G. Romero, M. G. Christiansen, A. Mohr, P. Anikeeva, Wireless magnetothermal deep brain stimulation. Science 347, 1477-1480 (2015).

16. K. L. Montgomery et al., Wirelessly powered, fully internal optogenetics for brain, spinal and peripheral circuits in mice. Nat Methods 12, 969-974 (2015).

17. M. J. Weber, A. Bhat, T. C. Chang, J. Charthad, A. Arbabian, in 2016 IEEE Topical Conference on Biomedical Wireless Technologies, Networks, and Sensing Systems (BioWireleSS), 2016. pp. 12-14.

18. A. Wickens et al., Magnetoelectric materials for miniature, wireless neural stimulation at therapeutic frequencies. bioRxiv, 461855 (2018).

19. J. Lee et al., in 2019 9th International IEEE/EMBS Conference on Neural Engineering (NER), 2019. pp. 871-874.

20. IEEE Standard for Safety Levels with Respect to Human Exposure to Electric, Magnetic, and Electromagnetic Fields, $0 \mathrm{~Hz}$ to $300 \mathrm{GHz}$. IEEE Std C95.1-2019 (Revision of IEEE Std C95.1-2005/Incorporates IEEE Std C95.1-2019/Cor 1-2019), 1-312 (2019).

21. C. Gabriel, S. Gabriel, E. Corthout, The dielectric properties of biological tissues: I. Literature survey. Phys Med Biol 41, 2231-2249 (1996).

22. P. S. Tofts, The distribution of induced currents in magnetic stimulation of the nervous system. Phys Med Biol 35, 1119-1128 (1990).

23. W. Klomjai, R. Katz, A. Lackmy-Vallee, Basic principles of transcranial magnetic stimulation (TMS) and repetitive TMS (rTMS). Ann Phys Rehabil Med 58, 208-213 (2015).

24. Z. D. Deng, S. H. Lisanby, A. V. Peterchev, Electric field depth-focality tradeoff in transcranial magnetic stimulation: simulation comparison of 50 coil designs. Brain Stimul 6, 1-13 (2013).

25. Y. Roth, A. Amir, Y. Levkovitz, A. Zangen, Three-dimensional distribution of the electric field induced in the brain by transcranial magnetic stimulation using figure- 8 and deep H-coils. Journal of Clinical Neurophysiology 24, 31-38 (2007).

26. R. Guduru et al., Magnetoelectric 'spin' on stimulating the brain. Nanomedicine 10, 20512061 (2015). 
27. V. Corral-Flores, D. Bueno-Baques, R. F. d. Ziolo, Synthesis and characterization of novel $\mathrm{CoFe}_{2} \mathrm{O}_{4}-\mathrm{BaTiO}_{3}$ multiferroic core-shell-type nanostructures. Acta Materialia 58, 764-769 (2010).

28. M. M. Vopson, Y. K. Fetisov, G. Caruntu, G. Srinivasan, Measurement Techniques of the Magneto-Electric Coupling in Multiferroics. Materials (Basel) 10, 963 (2017).

29. E. B. Montgomery, Jr., J. T. Gale, Mechanisms of action of deep brain stimulation(DBS). Neurosci Biobehav Rev 32, 388-407 (2008).

30. D. J. Lee, C. S. Lozano, R. F. Dallapiazza, A. M. Lozano, Current and future directions of deep brain stimulation for neurological and psychiatric disorders. J Neurosurg 131, 333-342 (2019).

31. A. Nambu, H. Tokuno, M. Takada, Functional significance of the cortico-subthalamopallidal 'hyperdirect' pathway. Neuroscience research 43, 111-117 (2002).

32. G. E. Alexander, M. D. Crutcher, M. R. DeLong, Basal ganglia-thalamocortical circuits: parallel substrates for motor, oculomotor, "prefrontal" and "limbic" functions. Prog Brain Res 85, 119-146 (1990).

33. J. I. Morgan, T. Curran, Role of ion flux in the control of c-fos expression. Nature 322, 552-555 (1986).

34. H. Davson, Physiology of the cerebrospinal fluid. (Churchill, London, 1967). 